Research paper

\title{
Automatic 3D aortic annulus sizing by computed tomography in the planning of transcatheter aortic valve implantation
}

\author{
Sandro Queirós a, b, c, *, Christophe Dubois ${ }^{\text {a, d }}$, Pedro Morais ${ }^{\text {a, b, e }}$, Tom Adriaenssens ${ }^{\text {a, d }}$ \\ Jaime C. Fonseca ${ }^{c}$, João L. Vilaça ${ }^{\text {b, f }}$, Jan D'hooge ${ }^{a}$ \\ a Dept. of Cardiovascular Sciences, KU Leuven, Leuven, Belgium \\ ${ }^{\mathrm{b}}$ ICVS/3B's - PT Government Associate Laboratory, Braga, Guimarães, Portugal \\ ${ }^{\mathrm{c}}$ Algoritmi Center, School of Engineering, University of Minho, Guimarães, Portugal \\ d Dept. of Cardiovascular Diseases, University Hospital Leuven, Leuven, Belgium \\ e INEGI, Faculty of Engineering, University of Porto, Porto, Portugal \\ ${ }^{\mathrm{f}}$ DIGARC - Polytechnic Institute of Cávado and Ave, Barcelos, Portugal
}

\section{A R T I C L E I N F O}

\section{Article history:}

Received 20 December 2016

Accepted 29 December 2016

Available online 30 December 2016

\section{Keywords:}

Computed tomography

TAVI

Aortic valve sizing

Fully automatic analysis

Semi-automatic analysis

\begin{abstract}
A B S T R A C T
Background: Accurate imaging assessment of aortic annulus (AoA) dimension is paramount to decide on the correct transcatheter heart valve (THV) size for patients undergoing transcatheter aortic valve implantation (TAVI). We evaluated the feasibility and accuracy of a novel automatic framework for multidetector row computed tomography (MDCT)-based TAVI planning.

Methods: Among 122 consecutive patients undergoing TAVI and retrospectively reviewed for this study, 104 patients with preoperative MDCT of sufficient quality were enrolled and analyzed with the proposed software. Fully automatic (FA) and semi-automatic (SA) AoA measurements were compared to manual measurements, with both automated and manual-based interobserver variability (IOV) being assessed. Finally, the effect of these measures on hypothetically selected THV size was evaluated against the implanted size, as well as with respect to manually-derived sizes.

Results: FA analysis was feasible in $92.3 \%$ of the cases, increasing to $100 \%$ if using the SA approach. Automatically-extracted measurements showed excellent agreement with manually-derived ones, with small biases and narrow limits of agreement, and comparable to the interobserver agreement. The SA approach presented a statistically lower IOV than manual analysis, showing the potential to reduce interobserver sizing disagreements. Moreover, the automated approaches displayed close agreement with the implanted sizes, similar to the ones obtained by the experts.

Conclusion: The proposed automatic framework provides an accurate and robust tool for AoA measurements and THV sizing in patients undergoing TAVI.

(C) 2016 Society of Cardiovascular Computed Tomography. Published by Elsevier Inc. All rights reserved.
\end{abstract}

\section{Introduction}

Although surgical aortic valve (AV) replacement (SAVR) remains the gold-standard treatment for symptomatic, severe aortic stenosis (AS), transcatheter AV implantation (TAVI) has become an alternative treatment option for high-risk/inoperable and, more

\footnotetext{
* Corresponding author. Life and Health Sciences Research Institute (ICVS), Universidade do Minho, Campus de Gualtar, 4710-057 Braga, Portugal.

E-mail addresses: sandroqueiros@med.uminho.pt (S. Queirós), christophe. dubois@uzleuven.be (C. Dubois), pedromorais@med.uminho.pt (P. Morais), tom. adriaenssens@uzleuven.be (T. Adriaenssens), jaime@dei.uminho.pt (J.C. Fonseca) jvilaca@ipca.pt (J.L. Vilaça), jan.dhooge@uzleuven.be (J. D'hooge).
}

recently, intermediate risk patients. ${ }^{1,2}$ While in SAVR, direct intraoperative sizing is viable for valve selection, sizing in TAVI must rely on preoperative imaging modalities. ${ }^{3-5}$ Accurate imaging assessment of aortic annulus (AoA) dimensions is thus paramount to decide on the correct transcatheter heart valve (THV) size, and can ultimately prevent procedural complications, such as paravalvular regurgitation, annular rupture or valve embolization. ${ }^{4-6}$

Currently available imaging modalities for TAVI planning include 2D/3D transthoracic (TTE) and transesophageal echocardiography (TEE), multidetector row computed tomography (MDCT) or even magnetic resonance imaging. ${ }^{3-6}$ Although 2D-TTE and 2DTEE were traditionally the standard, it is now generally accepted that 3D techniques are superior for such measurements, ${ }^{4,7}$ both due 
to the annulus' elliptical shape and due to the superior accuracy and reproducibility of area- and perimeter-based measures. ${ }^{4,7,8}$ Among the modalities with 3D capabilities, MDCT has been the preferred imaging technique, ${ }^{7,8}$ particularly thanks to its high reproducibility and superior image quality, ${ }^{3,9}$ with 3D-TEE being performed in more specialized centers.

Nonetheless, manual 3D analysis of either MDCT or TEE datasets is time-consuming and complex, being prone to both intra- and interobserver variability. ${ }^{10-12}$ Automatic analysis has the potential to shorten analysis time while mitigating the variability between experts, therefore increasing the reproducibility and accuracy of valve sizing. ${ }^{12,13}$ With this in mind, some commercial automated solutions have been presented for MDCT-based analysis. ${ }^{11,14-17}$ Nonetheless, most still require manual interaction, either to start the analysis or to correct its results, or present sub-optimal agreement with valve sizing performed by experts. ${ }^{11}$

Recently, we proposed a semi-automatic algorithm for AV segmentation in 3D-TEE datasets, ${ }^{13}$ which was later extended to a fully automatic framework for AV dimensioning in TAVI planning. ${ }^{18}$ The framework was shown to be accurate and robust, mitigating the variability associated with manual quantification and significantly reducing the required analysis time. Interestingly, and although originally presented for 3D-TEE processing, the framework has the potential to be applied to other 3D imaging modalities with minimal adaptations.

Since MDCT is often used within the TAVI workflow, in this study, we propose an adapted version of the original segmentation framework for fully automatic analysis of MDCT datasets. Moreover, we sought to evaluate its feasibility and accuracy for MDCT-based TAVI planning.

\section{Methods}

\subsection{Study population and TAVI procedure}

From June 2013 to August 2016, 122 consecutive high-risk or inoperable patients with symptomatic, severe AS underwent TAVI at the University Hospital Leuven, Leuven, Belgium, and were retrospectively reviewed for the present study. Of these, 5 valve-invalve procedures were excluded, together with another 13 cases due to inadequate pre-procedural MDCT image quality. Note that MDCT suitability was assessed by an expert cardiologist, being excluded when it prevented accurate manual analysis. As a result, 104 patients were enrolled in this study. All patients gave written informed consent before undergoing the TAVI procedure.

The clinical and echocardiographic characteristics of the study population are displayed in Table 1.

Prior to TAVI, all patients were evaluated by a team of expert cardiologists and cardiothoracic surgeons to determine their eligibility and plan the intervention. Patients underwent transfemoral $(82 / 104)$ or transapical (22/104) implantation of an Edwards SAPIEN XT or SAPIEN 3 THV (Edwards Lifesciences, California) of 23$\mathrm{mm}, 26-\mathrm{mm}$ or $29-\mathrm{mm}$ diameter. Valve size was chosen primarily based on AoA diameters derived from MDCT. In borderline cases (i.e. when dimensions were in-between the recommendations for 2 THV sizes), ${ }^{5}$ the team considered other clinical factors, such as gender, body size, amount and location of calcifications, and TEEderived AoA measurements. ${ }^{19}$ Overall, a SAPIEN XT was implanted in 58 patients (55.8\%), while the remaining 46 patients $(44.2 \%)$ received a SAPIEN 3 THV. Nineteen, 55 and 30 patients received a 23-mm, 26-mm and 29-mm THV, respectively. All implantations were successfully performed.

\subsection{Image acquisition}

Cardiac MDCT scans were performed using one of three multidetector scanners, including two dual-source scanners (Somatom Definition Flash and Somatom Force, Siemens Healthcare) and a single-source scanner (Aquilion ONE, Toshiba Medical Systems). All examinations included a non-ECG-synchronized acquisition of the entire aorta and iliofemoral arteries, and a prospective ECG-triggered scan of the heart, as recommended in the guidelines. ${ }^{10}$ Tube potential (80-120 kV) and current were determined based on body habitus. A timing bolus protocol was used to determine optimal contrast transit time. Data were subsequently acquired during an inspiratory breathhold in caudocranial direction. All images were reconstructed in the diastolic phase using a soft-tissue convolution kernel. Typical acquisition parameters can be found in Table 2. No beta-blockers were administered.

All patients enrolled in this study had a cMPR generated by an experienced radiologist at the time of TAVI planning using an automated aortic root analysis software (syngo.CT Cardiac Function - Valve Pilot and Vitrea) in three steps: (1) the data was imported into the offline workstation; (2) the software computed the aorta's centerline and extracted perpendicular cross-sectional image planes; (3) the cMPR was verified and, if needed, manually edited. Editing was deemed necessary if the centerline visibly deviated from the center of the lumen or if its orientation was oblique with regard to the true valve orientation.

\subsection{Manual analysis}

For each patient, the cMPR was viewed to identify the crosssectional image plane closest to the AoA plane, and was then manually delineated to extract annular area and area-derived diameter (given as $d_{\text {area }}=2 \times \sqrt{\text { Area } / \pi}$ ). ${ }^{5,10}$ In cases in which no cross-sectional image closely crossed the AoA, measurements were performed at the planes above and below the AoA, and their average computed. Fig. 1 presents some example tracings.

Table 1

Patients' baseline characteristics ( $\#=104)$.

\begin{tabular}{ll}
\hline Age (mean \pm SD) & $81.8 \pm 7.8$ \\
Male gender & $56(53.8 \%)$ \\
NYHA class $\geq$ III & $81(77.9 \%)$ \\
Surgical risk assessment & \\
STS score,\% (mean \pm SD) & $8.3 \pm 8.5$ \\
Logistic EuroSCORE,\% (mean \pm SD) & $28.0 \pm 16.2$ \\
EuroSCORE II,\% (mean \pm SD) & $11.2 \pm 9.1$ \\
Porcelain aorta & $20(19.2 \%)$ \\
Frailty & $27(26.0 \%)$ \\
Creatinine>2 mm/dL & $12(11.5 \%)$ \\
Cardiac risk factors & \\
Hypertension & $83(79.8 \%)$ \\
Diabetes & $22(21.2 \%)$ \\
Hyperlipidemia & $66(63.5 \%)$ \\
Past medical history & \\
Cardiac surgery & $39(37.5 \%)$ \\
Percutaneous coronary intervention & $40(38.5 \%)$ \\
Preexisting pacemaker/defibrillator & $13(12.5 \%)$ \\
Myocardial infarction & $20(19.2 \%)$ \\
Cardiomyopathy & $38(36.5 \%)$ \\
Stroke & $14(13.5 \%)$ \\
Cerebrovascular disease & $40(38.5 \%)$ \\
Peripheral vascular disease & $32(30.8 \%)$ \\
Chronic obstructive pulmonary disease & $26(25.0 \%)$ \\
Atrial fibrillation & $41(39.4 \%)$ \\
Clinical parameters & \\
Mean transaortic gradient, mmHg (mean \pm SD) & $44.6 \pm 16.0$ \\
Aortic valve area, cm ${ }^{2}$ (mean \pm SD) & $0.68 \pm 0.18$ \\
PAPmean, mmHg (mean \pm SD) & $31.5 \pm 10.2$ \\
EF,\% (mean \pm SD) & $49.9 \pm 12.0$ \\
\hline
\end{tabular}

Unless specified otherwise, values are $n$ (\%) of patients. 
Table 2

Typical scan parameters for the used MDCT scanners.

\begin{tabular}{|c|c|c|c|}
\hline Parameter & SOMATOM Definition Flash & SOMATOM Force & Aquilion ONE \\
\hline No. of patients & 76 & 27 & 1 \\
\hline Detector rows & $2 \times 128$ & $2 \times 192$ & 320 \\
\hline Detector row width (mm) & 0.6 & 0.6 & 0.5 \\
\hline Rotation time (ms) & 285 & 250 & 275 \\
\hline Contrast injection & $120 \mathrm{ml}$ at $5 \mathrm{ml} / \mathrm{s}$ & Patient-specific (38-50 ml at 2.5 to $1.5 \mathrm{ml} / \mathrm{s}$ ) & $120 \mathrm{ml}$ at $5 \mathrm{ml} / \mathrm{s}$ \\
\hline Reconstruction phase (R-R interval) & $60 \%$ & $65 \%$ & $75 \%$ \\
\hline Convolution kernel & B26f & Bv36d & FC08 \\
\hline Image resolution $\left(\mathrm{mm}^{3}\right)$ & $0.39 \times 0.39 \times 0.30$ & $0.38 \times 0.38 \times 0.30$ & $0.78 \times 0.78 \times 0.5$ \\
\hline Matrix size & $512 \times 512 \times 451$ & $512 \times 512 \times 551$ & $512 \times 512 \times 320$ \\
\hline
\end{tabular}

All manual delineations were performed by one experienced cardiologist (henceforth referred as M1), blinded to the automatic analysis and responsible for the measurements done at the time of TAVI planning. The same expert was also responsible for image quality assessment at the start of this study, and for excluding patients whose images were sub-optimal for manual analysis.

To determine interobserver reproducibility, a second expert cardiologist (referred as M2) reanalyzed all datasets, blinded to both manual and automatic analyses. M2 was free to choose different cross-sectional images for annular measurements if deemed necessary.

\subsection{Automatic analysis}

Automatic processing was performed using an adapted version of our recently proposed AV segmentation framework. ${ }^{13,18}$ Adaptations were made due to the different image appearance of the AV lumen and wall in MDCT and 3D-TEE images, as well as due to their different field-of-views (FOV).

In brief, the fully automatic framework (henceforth referred as FA) is divided into four main stages: (1) automatic AV detection and identification of its axis, which is given by one point at the ascending aorta (AscAo) and one at the left ventricular outflow tract (LVOT); (2) automatic segmentation of the AV tract wall; (3) automatic estimation of the short-axis (SAx) planes of interest in which to perform the clinical measurements, namely at the LVOT, AoA, sinuses of Valsalva (SoV) and sinotubular junction (STJ); (4) computation of the relevant measures (area, perimeter and diameters) at the identified SAx planes. The analysis workflow is shown in Fig. 2.

The first block uses the specific appearance of the AV wall and lumen to automatically detect them. Since the framework was originally developed for 3D-TEE images (smaller FOV than the MDCT one), an additional first step was added to identify an AV TEE-like FOV in the MDCT image (Fig. 2A-1). This process is achieved through a multi-atlas registration approach similar to the method describes by Kirişli et al., ${ }^{20}$ in which the target MDCT image is registered with 8 known atlases and their candidate $A V$ regions combined to estimate the AV region of interest (ROI). This step increases the robustness against different MDCT FOVs, while its result prepares the data for the previously developed initialization algorithm. ${ }^{18}$ This algorithm searches for the AV centroid within the identified ROI, which is then used to estimate the AV orientation. Subsequently, the leaflets' region is searched, and used to position both AscAo and LVOT points (defining the axis, Fig. 2A-2). Since in MDCT the AV lumen is brighter than its wall, all algorithms consider bright-to-dark image transitions (rather than dark-to-bright ones, like in 3D-TEE). Moreover, since in MDCT the leaflets' region can

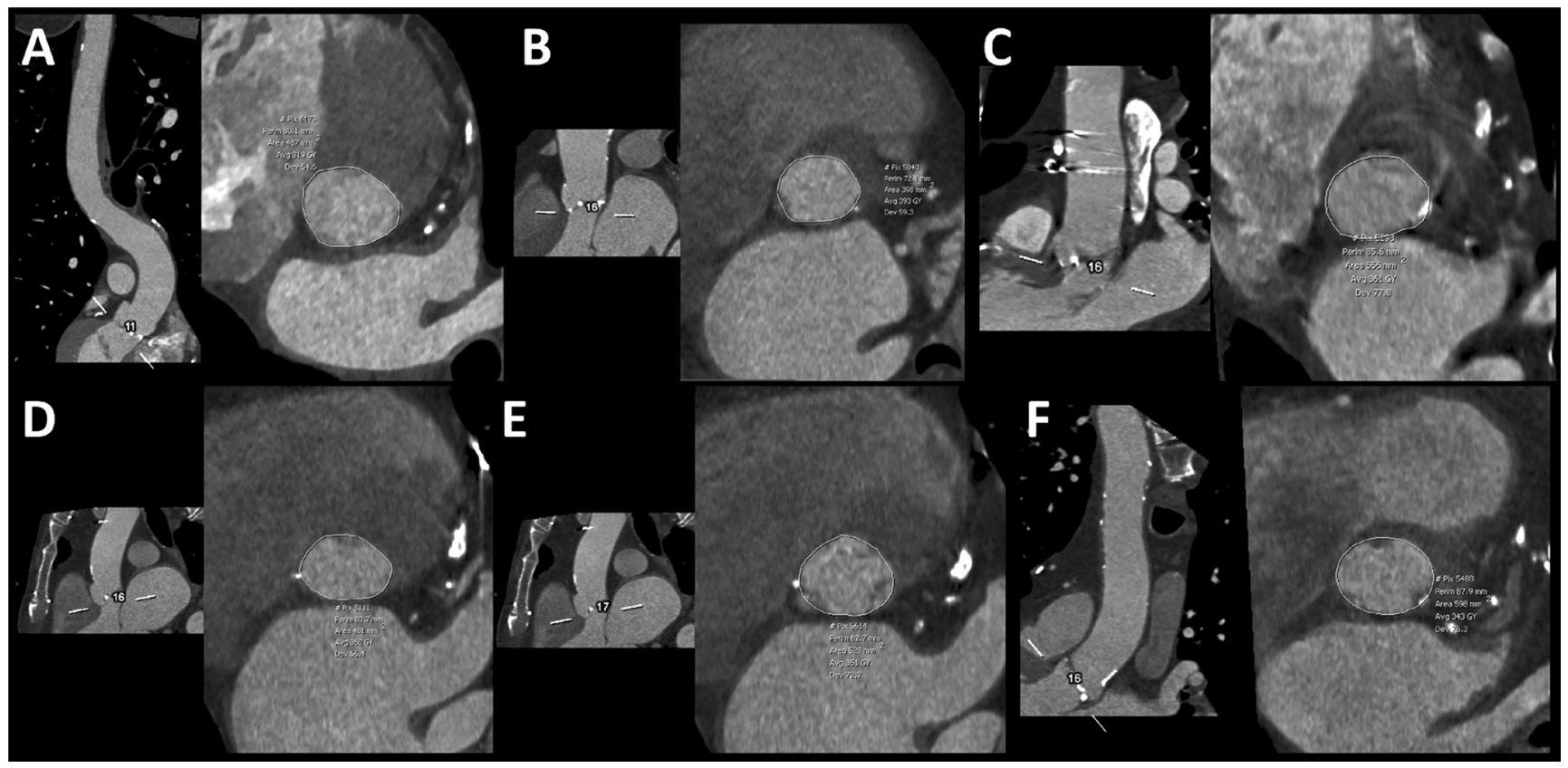

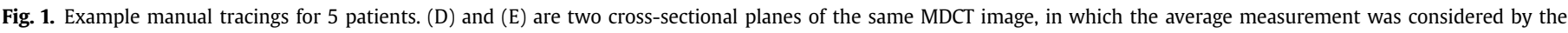
cardiologist expert. For $(A)-(C)$ and $(F)$, only one plane was delineated. 


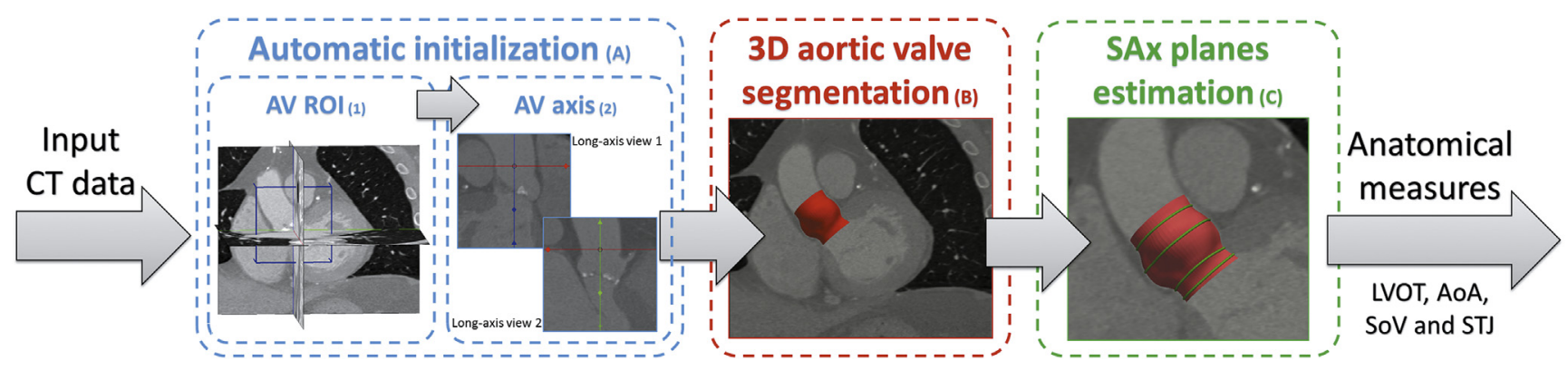

Fig. 2. Analysis workflow for the proposed fully automatic framework.

present darker (i.e., leaflet tissue) and brighter (i.e., calcifications) structures than the lumen, the leaflets' detection stage was modified to search for highly variable regions within the lumen (rather than bright structures only, as in TEE).

The second block uses our previously proposed shape-based segmentation strategy to accurately delineate the AV wall inbetween the two identified points (Fig. 2B). ${ }^{13}$ For this purpose, intensity and shape-based features are combined in an automatic border detection algorithm. Shape-prior information is included using a statistical shape model (i.e., model that statistically describes AV shape variability in the population). This information is embedded to guarantee that a valve-like shape is segmented, and thus complements the image information in potentially misleading regions. The model was created from manually delineated valves, using MDCT data of 40 patients not included in this study.

Finally, the third block estimates the SAx planes' position by aligning the target AV segmentation with reference AV surfaces (in which these planes were previously identified). ${ }^{18}$ By performing this shape-based alignment with known references, the planes' location and orientation can be robustly estimated for the new image (Fig. 2C). By cutting the segmented surface at each SAx plane, the relevant measures can be extracted. In the present study, only the measures at the AoA plane will be assessed.

In order to understand the influence of the automatic AV identification, a semi-automatic version of the algorithm was also analyzed (henceforth referred as SA). In this case, the only difference is that the identification of the AV axis is performed manually by an observer through the input of two points in the AV lumen: one at the AscAo and another at the LVOT. Then, the remaining 3 stages $\left(2^{\text {nd }}\right.$ and $3^{\text {rd }}$ block, plus measurements extraction) are automatically performed. This input mitigates the possibility of AV misidentification or sub-optimal positioning. To assess the reproducibility of the SA approach, a second observer repeated the analysis (i.e. manually identified the two points required to initialize the automatic processing), blinded to the first observer's results.

Further technical details on these algorithms can be found in the original publications. ${ }^{13,18}$

\subsection{Prosthesis size selection}

For each patient and each measuring approach, the theoretical prosthesis size was identified. Manufacturer-recommended MDCTbased sizing algorithms were considered for each THV model., Note that manufacturer recommendations present an overlap between different THV model sizes, allowing to over- or undersize according to patient-specific clinical characteristics. ${ }^{5,19}$ In this sense, borderline cases (i.e., whose measures lay in the overlapping regions) were also considered. Fig. 3 summarizes the valve selection strategy used for each THV model.

\subsection{Statistical analysis}

To quantify the applicability of the proposed fully automatic framework, feasibility was assessed by one observer by considering the analysis successful when the detected and delineated object roughly spanned the AV tract from the AscAo to the LVOT. In other words, if the automatic initialization step failed, upon visual inspection, to roughly estimate the position of these two points in the MDCT image, the analysis was considered incorrect. Note that results for the FA approach exclusively include successfully analyzed datasets.

The accuracy of the automatic area-derived diameters was assessed by comparing against manually determined reference values. The measured diameters are given as mean \pm standard deviation (SD) for each approach. To assess agreement, BlandAltman (BA) analyses were performed between the FA and SA approaches and each expert's measures, computing biases and limits of agreement (LOAs, i.e. 1.96*SD). The statistical significance of the biases was tested using a two-tailed paired $t$-test against zero. The same analysis was performed between the experts' manual measures to assess interobserver variability (IOV). To quantify the reproducibility of the SA approach, the agreement between both observers' SA analyses was also evaluated. To compare the LOAs of the automatic approaches and the IOV, a two-tailed F-test was used. All analyses considered a $\mathrm{p}<0.05$ as statistically significant. For completeness sake, the intraclass correlation coefficient (ICC) between approaches, and its 95\% confidence interval (CI), was also computed.

To assess the clinical significance of the extracted measures for TAVI planning, the effect on the hypothetically selected THV size was evaluated and compared to the implanted one. Any borderline case was considered successfully sized if either one of the possible THV sizes was the implanted one. Such strategy is closely related to the sizing rules applied by the TAVI team during the original planning, as any borderline case was, at the time, decided based on additional features. This analysis against the implanted size was performed independently for both automatic approaches, as well as for each expert's measures. Moreover, the sizing agreement between each pair of approaches was also assessed. Such comparison allows understanding the actual effect of the differences between measures, disregarding the TAVI team's specific decisions in the original planning. Once again, any borderline case was considered successfully sized if it matches the other approach's size (or sizes, if in the overlapping region).

\section{Results}

\subsection{Feasibility study}

The fully automatic analysis was found to be feasible in $92 \%$ of cases (96/104). Of the 8 incorrectly initialized cases, 2 were related 


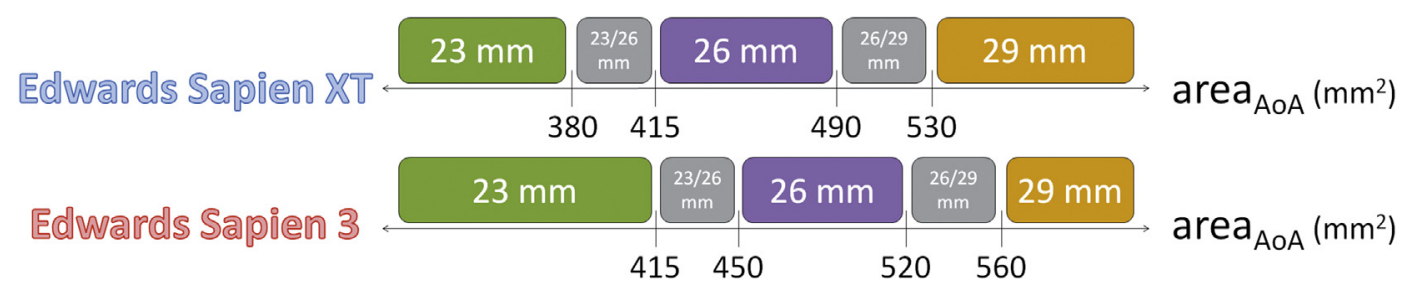

Fig. 3. Valve selection strategy used for each THV model, based on the measured AoA area.

to a sub-optimal axis orientation estimation, 5 to the LVOT positioning and 1 to the AscAo positioning. Semi-automatic analysis was feasible in all cases.

\subsection{Comparison of AoA measurements}

Table 3 presents both automatically and manually area-derived diameters, and summarizes the agreement between approaches. Manual IOV is also depicted. Fig. 4 illustrates the associated BlandAltman plots. Both automatic approaches presented excellent agreement (ICC $\geq 0.89$ ) against both experts' measures, with their LOAs being as narrow as the IOV ones $(S D<1 \mathrm{~mm})$. Interestingly, the SA approach was found to be highly reproducible (BA:$0.12 \pm 0.53$; ICC:0.98 [CI:0.95-0.99]; Fig. 4F), showing its robustness with respect to the user-defined points. In fact, IOV for the semi-automatic approach was significantly lower than the IOV for the manual analysis. Finally, no statistically significant difference was found between both automatic approaches (FA and SA). Fig. 5 illustrates 8 representative fully automatic segmentation results.

\subsection{Valve sizing}

The distribution of THV size selection for each approach is illustrated in Fig. 6. To allow comparison between methods, unsuccessfully analyzed cases for the FA method are depicted in the associated implanted size. No size-related tendency was found for these failure cases.

The THV sizing agreement for each approach against the implanted one is given in Table 4. Interestingly, the semi-automatic approach showed the best agreement. Although no tendency was generally found for the second expert, both first expert and automated approaches presented most disagreements as undersized THVs.

When evaluating between measurement approaches (vs. M1 and M2), the sizing agreements were better for almost all cases (except FA vs. M2) than when compared against the implanted sizes. Again, the SA approach outperformed the FA method. When assessing disagreements, the second expert showed a tendency to oversize compared to all other approaches, with no tendency found among these.

\section{Discussion}

In our single-center TAVI population, the proposed fully automatic framework was feasible in $92 \%$ of the cases, with only 8 cases failing due to sub-optimal detection of anatomic structures. Notwithstanding, if the user provided the initialization (i.e. the SA approach), the method was feasible in all cases. Although being a single-center retrospective study, the population included most of the patient variability frequently found in a TAVI population, such as patients with severe valve calcifications, previously implanted mitral valve prosthesis and/or pacemakers/defibrillators. The latter cases are overly burdensome, particularly for successful automatic initialization, due to the artifacts caused on the MDCT image. Moreover, the method was feasible for MDCT images with variable FOVs and from multiple scanners, which is primarily linked to the robustness of the multi-atlas initialization step.

In order to minimize the rate of procedural complications associated with incorrect prosthesis sizing, accurate AV measurements are crucial. Manual delineation is challenging, time consuming and operator-dependent, which hamper an accurate and reproducible pre-TAVI analysis. The proposed automatic tool aims at mitigating these problems, by providing accurate and reproducible measures. When compared to expert measurements, the automatic approaches showed an excellent agreement with small biases and narrow LOAs. Among them, the semi-automatic approach showed a tendency for a better agreement (although not statistically significant). This improvement is mainly related to a small number of cases in which the FA approach presented a visually correct initialization, but with a sub-optimal AV axis' positioning (by positioning one point close to the wall). Although the segmentation and subsequent analysis can still be successfully performed, the obtained measures are slightly affected by the initial sub-optimal axis positioning. By providing these points in the SA approach, a better delineation is achieved and a better agreement is obtained. Notwithstanding, both approaches presented competitive results with respect to the experts' IOV.

Given the manual analysis' dependence on the observer's experience, automatic tools offer the possibility to reduce intraand interobserver variability. ${ }^{16,21}$ Indeed, the SA approach was shown to be highly reproducible, with the variability between measurements performed by two observers statistically lower than

Table 3

Comparison of area-derived diameters measured by automatic and manual approaches.

\begin{tabular}{|c|c|c|c|c|c|c|}
\hline \multirow[t]{2}{*}{ Method } & \multirow[t]{2}{*}{ \# } & \multirow[t]{2}{*}{$\boldsymbol{d}_{\text {area }}(\mathrm{mm})$} & \multicolumn{2}{|l|}{ vs.M1 } & \multicolumn{2}{|l|}{ vs.M2 } \\
\hline & & & $\mathrm{BA}$ & ICC (95\% CI) & $\overline{\mathrm{BA}}$ & ICC $(95 \%$ CI $)$ \\
\hline M1 & 104 & $24.6 \pm 2.11$ & - & - & $-0.35^{a} \pm 0.70$ & $0.93(0.87-0.96)$ \\
\hline M2 & 104 & $24.9 \pm 2.16$ & $0.35^{\mathrm{a}} \pm 0.70$ & $0.93(0.87-0.96)$ & - & - \\
\hline SA & 104 & $24.5 \pm 2.26$ & $-0.01 \pm 0.79$ & $0.93(0.90-0.95)$ & $-0.36^{\mathrm{a}} \pm 0.91^{\mathrm{b}}$ & $0.90(0.84-0.94)$ \\
\hline FA & 96 & $24.7 \pm 1.98$ & $0.08 \pm 0.81$ & $0.92(0.88-0.95)$ & $-0.25^{\mathrm{a}} \pm 0.97^{\mathrm{b}}$ & $0.89(0.83-0.92)$ \\
\hline
\end{tabular}

Bland-Altman (BA) analyses are given as bias $\pm 1.0^{*} \mathrm{SD}$

a $\mathrm{p}<0.05$, two-tailed paired $t$-test against zero.

b $\mathrm{p}<0.05$, two-tailed F-test against interobserver variability (M1 vs. M2). 

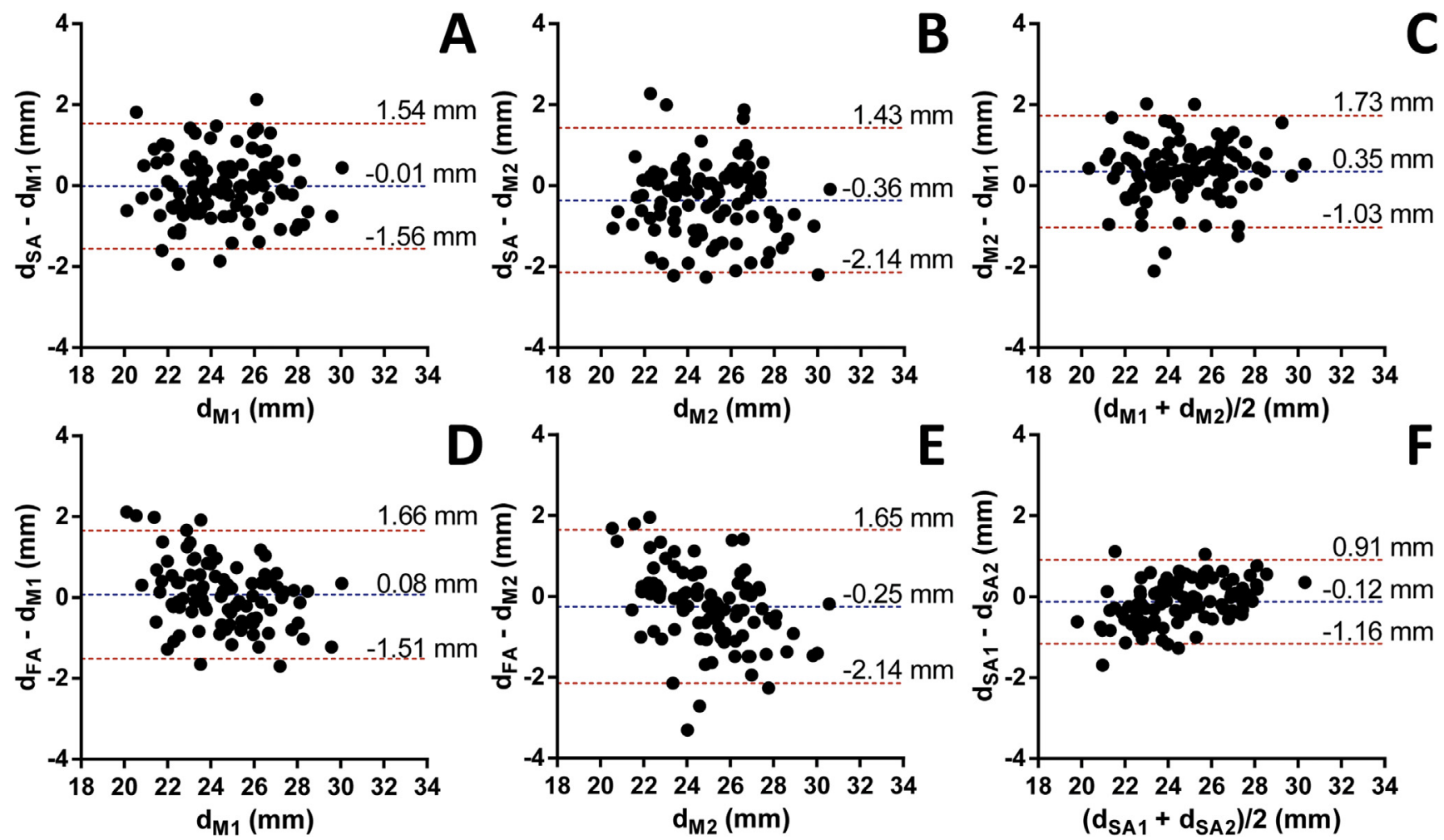

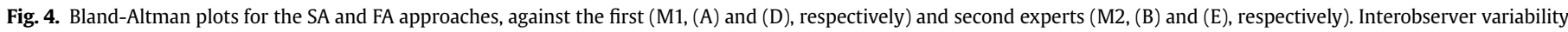

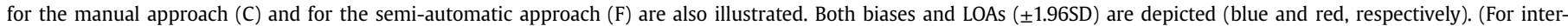
pretation of the references to colour in this figure legend, the reader is referred to the web version of this article.)

when using the manual procedure. In the latter case, expert experience influences the MPR slice (or slices) chosen as the AoA plane, as well as the technique used to delineate the AV lumen. Importantly, notice that a cMPR was used in our study for the manual analysis, which already tends to present a lower IOV compared to the more traditional double-oblique reconstruction technique (which additionally requires manual alignment and reconstruction of the AoA plane directly in the 3D MDCT image). ${ }^{22}$ Such a result emphasizes the increased reproducibility obtained when using the proposed automated framework, in which the user

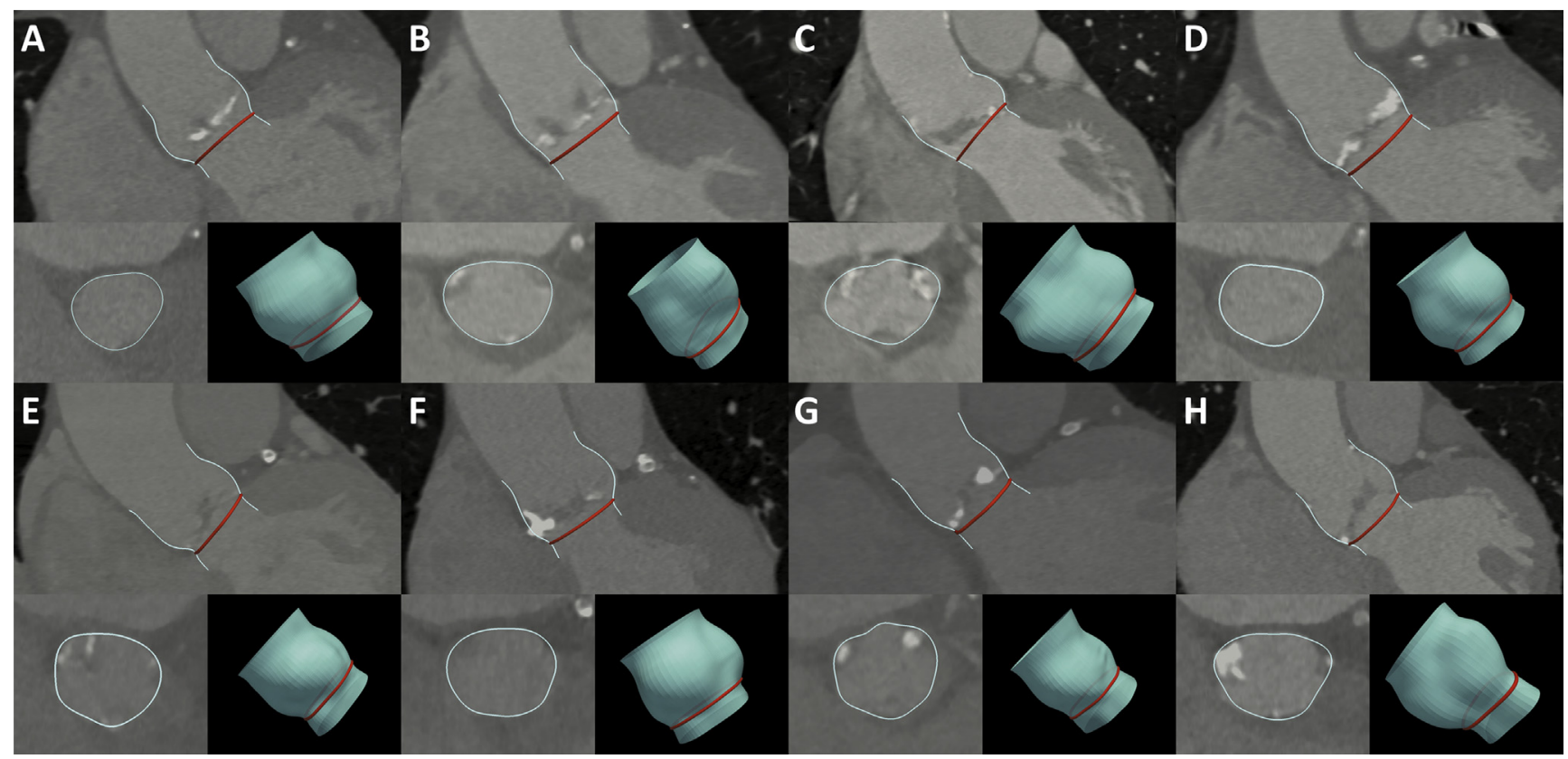

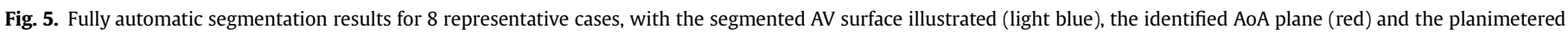
tracing. (For interpretation of the references to colour in this figure legend, the reader is referred to the web version of this article.) 


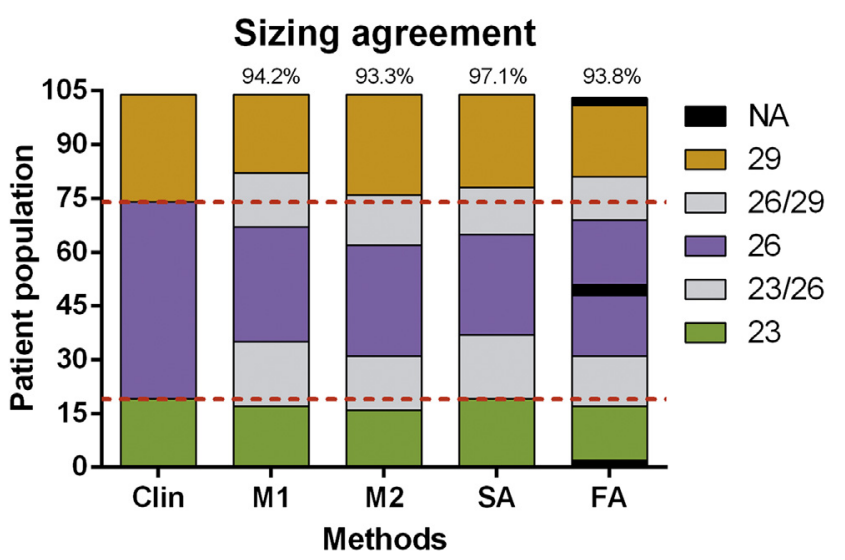

Fig. 6. Distribution of THV size theoretically selected by each measuring approach, as well as the true implanted size (Clin). The sizing agreement is shown at the top of each bar. Unsuccessfully analyzed cases for the FA method are also depicted within the associated implanted size (black).

only identifies two points and the software automatically segments and identifies the AoA plane. In the case of a fully automatic analysis, no interaction is required at all, therefore eliminating any userinduced variability.

When compared to state-of-the-art and commercial solutions, the proposed framework presented competitive results, both in terms of feasibility ${ }^{11}$ and measurements agreement. ${ }^{11,14-17,21,23,24}$ In fact, the LOAs against expert measurements were lower than many reported in the literature for automated solutions. ${ }^{17,21,23,24}$ Interestingly, when assessing interobserver variability for the SA approach, LOAs for the proposed software were also excellent (BA:0.02 \pm 0.70 in Ref.15, and ICC: 0.98 in Ref. ${ }^{21}$ ). Such result is true even against other reported IOV for manual analysis. ${ }^{15,19,21}$ Note that these studies presented IOV results comparable to the ones reported in our study (LOAs with $0.6-0.8 \mathrm{~mm}$ of SD, and ICC between 0.87 and 0.95 , are found in the literature).

Regarding the hypothetically selected THV size, the automatic approaches (FA and SA) presented an excellent agreement towards the implanted sizes (97\% and 94\% agreement, respectively). Such rates of agreement were similar to the ones obtained by the experts (M1 and M2 vs. implanted one, 94\%), demonstrating the interchangeability of automatically-extracted measurements for preTAVI sizing. With the exception of the $2^{\text {nd }}$ expert, the remaining cases (automatic ones and $1^{\text {st }}$ expert) presented most (or all) of the disagreements as undersized THVs. Nonetheless, the comparison against the actual implanted sizes is influenced by the TAVI team's specific decisions during TAVI, which are linked to their experience at the time, as well as to the time-variable accepted clinical practices. Indeed, if compared to the manually-sized THVs (vs. M1 and M2, Table 4), the automatic approaches presented even higher rates of agreement (up to 100\%). Once again, these results were similar to the agreement found between experts (98\%). Moreover, the sizing agreement were very competitive to the ones reported in other similar studies (between 80 and $90 \%^{9,11,25}$ ). In fact, compared to the results reported for a fully automatic approach, ${ }^{11}$ a considerably better agreement against manual-based THV sizing was obtained. Finally, no correlation was found between disagreements and THV model and/or size, suggesting that the proposed approach is suitable for the general TAVI population. Moreover, although no assessment was performed with regard to the degree of valve calcification, the study population included both moderate and severe calcification cases, showing once again the software's aptness for THV sizing in the clinical practice.

Notably, even though this study focused only on the accuracy of the AoA measurements, the framework is able to extract measurements at 3 other levels, namely LVOT, SoV and STJ. These are also relevant for the preoperative planning, ${ }^{4,12}$ being required for the sizing of some THV models. ${ }^{4}$ Note that their accuracy is linked to the one obtained for the AoA (i.e. the same algorithm is used), and was demonstrated in the original publication. ${ }^{13}$

The automatic extraction of additional measurements (e.g., distance from AoA to coronary ostia or leaflets' tips) is focus of future research. Nonetheless, the framework is currently integrated in a custom non-commercial software, Speqle 3D, in which the user can already manually identify additional relevant landmarks (e.g., ostia or leaflets' tips) and additional measurements are extracted (i.e. it automatically computes the distance from the user-identified landmark and the automatically estimated AoA plane). Moreover, the entire framework allows a supervised analysis (i.e. with small manual corrections), which might be relevant in case of suboptimal analysis in particularly abnormal anatomical characteristics. Nevertheless, neither feature was validated in the current study.

\section{Conclusions}

In summary, this study demonstrates that the proposed automatic MDCT processing software provides a reliable and robust tool for AoA measurements and THV sizing in patients undergoing TAVI. The fully automatic framework was found to be feasible in $92.3 \%$ of our single-center study population, increasing to $100 \%$ if using the semi-automatic approach. Importantly, automatically-extracted measurements showed excellent agreement with manuallyderived ones, ultimately leading to an excellent sizing agreement with implanted THVs. Such rate of agreement was similar to the ones obtained by the experts, thus proving the interchangeability of using the automatically-extracted measurements for pre-TAVI sizing and showing the appeal of the proposed software for clinical practice.

\section{Conflict of interest}

There is no conflict of interest to declare.

Table 4

Frequency of (dis)agreement on THV size against implanted one and between methods.

\begin{tabular}{|c|c|c|c|c|c|c|c|c|c|c|}
\hline \multirow[t]{2}{*}{ Method } & \multirow[t]{2}{*}{$\#$} & \multicolumn{3}{|c|}{ vs. Implanted } & \multicolumn{3}{|l|}{ vs.M1 } & \multicolumn{3}{|l|}{ vs.M2 } \\
\hline & & Equal & UE & $\mathrm{OE}$ & Equal & UE & $\mathrm{OE}$ & Equal & UE & $\mathrm{OE}$ \\
\hline M1 & 104 & $98(94 \%)$ & $5(5 \%)$ & $1(1 \%)$ & - & - & - & $102(98 \%)$ & $2(2 \%)$ & $0(0 \%)$ \\
\hline M2 & 104 & $97(93 \%)$ & $3(3 \%)$ & $4(4 \%)$ & 102(98\%) & $0(0 \%)$ & $2(2 \%)$ & - & - & - \\
\hline SA & 104 & $101(97 \%)$ & $3(3 \%)$ & $0(0 \%)$ & $104(100 \%)$ & $0(0 \%)$ & $0(0 \%)$ & 101(97\%) & $3(3 \%)$ & $0(0 \%)$ \\
\hline FA & 96 & $90(94 \%)$ & $4(4 \%)$ & $2(2 \%)$ & $93(97 \%)$ & $2(2 \%)$ & $1(1 \%)$ & 89(93\%) & $5(5 \%)$ & $2(2 \%)$ \\
\hline
\end{tabular}

OE: overestimation; UE: underestimation.

Results given as number of cases (percentage of total) of (dis)agreement. 


\section{Acknowledgements}

The authors acknowledge funding support from FCT - Fundação para a Ciência e a Tecnologia, Portugal, and the European Social Found, European Union, through the Programa Operacional Capital Humano $(\mathrm{POCH})$ in the scope of the PhD grants SFRH/BD/93443/ 2013 (S. Queirós) and SFRH/BD/95438/2013 (P. Morais), and the project 'PersonalizedNOS (01-0145-FEDER-000013)' co-funded by Programa Operacional Regional do Norte (QREN), through Fundo Europeu de Desenvolvimento Regional (FEDER).

\section{References}

1. Leon MB, Smith CR, Mack M, et al. Transcatheter aortic-valve implantation for aortic stenosis in patients who cannot undergo surgery. N. Engl J Med. 2010;363:1597-1607.

2. Leon MB, Smith CR, Mack MJ, et al. Transcatheter or surgical aortic-valve replacement in intermediate-risk patients. N. Engl J Med. 2016;374:1609-1620.

3. Bloomfield GS, Gillam LD, Hahn RT, et al. A practical guide to multimodality imaging of transcatheter aortic valve replacement. JACC Cardiovasc Imaging. 2012;5:441-455

4. Zamorano J, Gonçalves A, Lancellotti P, et al. The use of imaging in new transcatheter interventions: an EACVI review paper. Eur Heart J Cardiovasc Imaging. 2016;17:835, 835af.

5. Kasel AM, Cassese S, Bleiziffer S, et al. Standardized imaging for aortic annular sizing: implications for transcatheter valve selection. JACC Cardiovasc Imaging. 2013:6:249-262.

6. Kenny C, Monaghan M. How to assess aortic annular size before transcatheter aortic valve implantation (TAVI): the role of echocardiography compared with other imaging modalities. Heart. 2014.

7. Figulla HR, Webb JG, Lauten A, Feldman T. The transcatheter valve technology pipeline for treatment of adult valvular heart disease. Eur Heart J. 2016.

8. Bax JJ, Delgado V, Bapat V, et al. Open issues in transcatheter aortic valve implantation. Part 1: patient selection and treatment strategy for transcatheter aortic valve implantation. Eur Heart J. 2014;35:2627-2638.

9. Schmidkonz C, Marwan M, Klinghammer L, et al. Interobserver variability of CT angiography for evaluation of aortic annulus dimensions prior to transcatheter aortic valve implantation (TAVI). Eur J Radiology. 2014;83:1672-1678.

10. Achenbach S, Delgado V, Hausleiter J, Schoenhagen P, Min JK, Leipsic JA. SCCT expert consensus document on computed tomography imaging before transcatheter aortic valve implantation (TAVI)/transcatheter aortic valve replacement (TAVR). J Cardiovasc Comput Tomogr. 2012;6:366-380.

11. Lou J, Obuchowski NA, Krishnaswamy A, et al. Manual, semiautomated, and fully automated measurement of the aortic annulus for planning of transcatheter aortic valve replacement (TAVR/TAVI): analysis of interchangeability. J Cardiovasc Comput Tomogr. 2015;9:42-49.
12. Hahn RT, Little SH, Monaghan MJ, et al. Recommendations for comprehensive intraprocedural echocardiographic imaging during TAVR. JACC Cardiovasc Imaging. 2015;8:261-287.

13. Queirós S, Papachristidis A, Barbosa D, et al. Aortic valve tract segmentation from 3D-TEE using shape-based B-spline Explicit Active Surfaces. IEEE Trans Med Imaging. 2016;35:2015-2026.

14. Calleja A, Thavendiranathan P, Ionasec RI, et al. Automated quantitative 3 dimensional modeling of the aortic valve and root by 3-dimensional transesophageal echocardiography in normals, aortic regurgitation, and aortic stenosis comparison to computed tomography in normals and clinical implications. Circ Cardiovasc Imaging. 2013;6:99-108.

15. Foldyna B, Jungert C, Luecke C, et al. CT evaluation prior to transapical aortic valve replacement: semi-automatic versus manual image segmentation. Int I Cardiovasc Imaging. 2015;31:1233-1242.

16. Delgado V, Ng AC, Schuijf JD, et al. Automated assessment of the aortic root dimensions with multidetector row computed tomography. Ann Thorac Surg. 2011;91:716-723.

17. Stortecky S, Heg DH, Gloekler S, Wenaweser PM, Windecker S, Buellesfeld L. Accuracy and reproducibility of aortic annulus sizing using a dedicated threedimensional computed tomography reconstruction tool in patients evaluated for transcatheter aortic valve replacement. EuroIntervention. 2014;10: 339-346.

18. Queirós S, Papachristidis A, Morais P, et al. Fully automatic 3D-TEE segmentation for the planning of transcatheter aortic valve implantation. in press IEEE Trans Biomed Eng. 2016.

19. Husser O, Holzamer A, Resch M, et al. Prosthesis sizing for transcatheter aortic valve implantation-comparison of three dimensional transesophageal echocardiography with multislice computed tomography. Int J Cardiol. 2013;168: 3431-3438.

20. Kirișli H, Schaap M, Klein S, et al. Evaluation of a multi-atlas based method for segmentation of cardiac CTA data: a large-scale, multicenter, and multivendor study. Med Phys. 2010;37:6279-6291.

21. Watanabe Y, Morice M-C, Bouvier E, et al. Automated 3-dimensional aortic annular assessment by multidetector computed tomography in transcatheter aortic valve implantation. JACC Cardiovasc Interv. 2013;6:955-964.

22. Agarwal PP, Liu PS, Hagan P, Booher AM, Watcharotone K, Quint LE. Proximal thoracic aortic diameters: transesophageal-echocardiography-versus computed-tomography-derived measurements. Clin Imaging. 2016;40: $191-199$.

23. Elattar M, Wiegerinck E, van Kesteren F, et al. Automatic aortic root landmark detection in CTA images for preprocedural planning of transcatheter aortic valve implantation. Int J Cardiovasc imaging. 2016;32:501-511.

24. Swee JK, Grbić S. Advanced Transcatheter Aortic Valve Implantation (TAVI) Planning from CT with ShapeForest. Medical Image Computing and Computerassisted Intervention-MICCAI 2014. Springer; 2014:17-24.

25. Kempfert J, Van Linden A, Lehmkuhl L, et al. Aortic annulus sizing: echocardiographic versus computed tomography derived measurements in comparison with direct surgical sizing. Eur J Cardio-Thoracic Surg. 2012;42:627-633. 